\title{
Serum choline is associated with hepatocellular carcinoma survival: a prospective cohort study
}

\author{
Zhao-Yan Liu', Dinuerguli Yishake', Ai-Ping Fang ${ }^{1}$, Dao-Ming Zhang ${ }^{1}$, Gong-Cheng Liao ${ }^{1}$, Xu-Ying Tan', \\ Yao-Jun Zhang ${ }^{2}$ and Hui-Lian Zhu ${ }^{1 *}$
}

\begin{abstract}
Background: Higher choline and betaine levels have been linked to lower risk of liver cancer, whereas existing data in relation to hepatocellular carcinoma (HCC) prognosis are scarce. Our objective was to examine the associations of the serum choline and betaine with HCC survival.

Methods: 866 newly diagnosed HCC patients were enrolled in the Guangdong Liver Cancer Cohort. Serum choline and betaine were assessed using high-performance liquid chromatography with online electro-spray ionization tandem mass spectrometry. Liver cancer-specific survival (LCSS) and overall survival (OS) were calculated. Cox proportional hazards models were used to estimate the hazard ratios (HRs) and 95\% confidence intervals (Cls).

Results: Serum choline levels were associated with better LCSS (T3 vs. T1: HR $=0.69,95 \%$ Cl: $0.51-0.94 ; P_{\text {-trend }}<0.05$ ) and OS (T3 vs. T1: HR $=0.73,95 \% \mathrm{Cl}: 0.54-0.99 ; P_{\text {-trend }}<0.05$ ). The associations were significantly modified by C-reactive protein (CRP) levels but not by other selected prognostic factors including sex, age, etc. The favorable associations between serum choline and LCSS and OS were only existed among patients with CRP $\geq 3.0 \mathrm{mg} / \mathrm{L}$. No significant associations were found between serum betaine levels and either LCSS or OS.

Conclusions: This study revealed that higher serum choline levels were associated with better HCC survival, especially in HCC patients with systemic inflammation status. No significant associations were found between serum betaine and HCC survival. Our findings suggest the benefits of choline on HCC survival.
\end{abstract}

Trial registration: The Guangdong Liver Cancer Cohort was registered at clinicaltrials.gov as NCT03297255.

Keywords: Choline, Betaine, Hepatocellular carcinoma, Survival

\section{Background}

Hepatocellular carcinoma ( $\mathrm{HCC})$ is one of the most common malignancy, and is a leading cause of cancer-related morbidity and mortality worldwide [1]. Multiple wellestablished ways aimed for curative treatment of HCC patients. However, the prognosis is still poor with five-year

\footnotetext{
* Correspondence: zhuhl@mail.sysu.edu.cn

'Guangdong Provincial Key Laboratory of Food, Nutrition and Health, School of Public Health, Sun Yat-sen University, Guangzhou 510080, People's Republic of China

Full list of author information is available at the end of the article
}

survival in the range of $5-30 \%[1,2]$. To improve longterm survival and diminish suffering of HCC patients, many convenient modifiable ways have been proposed to guide patient care, among which favorable dietary nutrients and nutritional status $[3,4]$ remain as major challenges.

Choline is an essential nutrient present in all cells, and plays a wide range of physiological functions in the body [5]. For example, choline is important to maintain the structural integrity of cellular membranes by serving as a component of phosphatidylcholine, which comprises almost half of the cellular membranes [6]. And it is needed

C C The Author(s). 2020 Open Access This article is licensed under a Creative Commons Attribution 4.0 International License, which permits use, sharing, adaptation, distribution and reproduction in any medium or format, as long as you give appropriate credit to the original author(s) and the source, provide a link to the Creative Commons licence, and indicate if changes were made. The images or other third party material in this article are included in the article's Creative Commons licence, unless indicated otherwise in a credit line to the material. If material is not included in the article's Creative Commons licence and your intended use is not permitted by statutory regulation or exceeds the permitted use, you will need to obtain permission directly from the copyright holder. To view a copy of this licence, visit http://creativecommons.org/licenses/by/4.0/. The Creative Commons Public Domain Dedication waiver (http://creativecommons.org/publicdomain/zero/1.0/) applies to the data made available in this article, unless otherwise stated in a credit line to the data. 
to form acetylcholine, an important signaling neurotransmitter [6]. In addition, choline occupies critical roles in maintaining normal liver function. Particularly, choline is a major source of methyl groups via oxidation to betaine, their functions involving the re-methylation of homocysteine to methionine are critical for DNA and histone methylation homeostasis, and therefore have been reported to be associated with cancer risk [2] (including liver cancer) and survival [7-10]. Experimental studies have reported that when deprived of choline, varying degrees of liver damage and liver diseases developed, including elevated transaminases [11], affected lipid metabolism and transport [12], fatty liver [13], liver cirrhosis [13] and even liver cancer [14], whereas choline or betaine supplementation ameliorated liver damage [15, 16], and choline supplementation increased global DNA methylation and DNA methyltransferase expression in HepG2 cells [17], which implied that choline or betaine might not only be associated with hepatocarcinogenesis, but also with liver cancer survival. To date, although scarcely, three case control studies [18-20] have evaluated the relationship between choline and betaine and human liver cancer risk, in which consistently favorable effects of choline were found. Of note, previous studies reported seriously insufficient dietary choline intake in Chinese population [20, 21], and approximately $50 \%$ of the total HCC cases occurred in China [19]. No existing studies have reported the associations between choline and betaine and liver cancer survival, it would be a very meaningful topic to investigate the influence of choline and betaine status on HCC prognosis in Chinese population.

In this study, we aimed to examine serum choline and betaine in relation to HCC survival in the Guangdong Liver Cancer Cohort (GLCC), to testify the hypothesis that choline and betaine status would be associated with HCC survival.

\section{Methods}

\section{Study population}

GLCC is an ongoing prospective cohort study conducted at Sun Yat-sen University Cancer Center (SYSUCC), the detailed protocols have been described previously [22, 23]. The present analyses included adult HCC patients enrolled between September 2013 and February 2017 if they met all the following criteria: 1) diagnosed within 1 month, 2) had no history of other carcinoma or any anticancer therapy, 3) had donated available fasting blood samples. HCC was diagnosed by either imaging or histopathology based on National Comprehensive Cancer Network Clinical Practice Guidelines in Oncology: Hepatobiliary Cancers [24].

\section{Serum choline and betaine measurement}

Fasting blood samples were collected as soon as possible once recruited before any anticancer therapies. Serum concentrations of choline and betaine were assessed by high-performance liquid chromatography with online electro-spray ionization tandem mass spectrometry to measure the concentrations of choline, and betaine. Detailed protocol of choline measurement has been described previously [19]. Detection of serum betaine was similar to choline, except for the internal standard was d9betaine (obtained from Sigma-Aldrich, St. Louis, USA). The coefficients of variation for the between-run assays were 4.91 and $6.21 \%$ for choline and betaine, respectively.

\section{Survival data collection}

The date of recruitment was used as the start date of follow-up for GLCC patients, and the last outcome follow-up of the present study was conducted on September 26, 2017. We assessed liver cancer-specific survival (LCSS) and overall survival (OS), and deaths from liver cancer or any cause were assigned as outcome event of LCSS or OS, respectively. The ascertainment process of deaths has been described previously $[22,23]$.

\section{Other covariates}

A face-to-face structured questionnaire was used to collect sociodemographic and lifestyle data by well-trained research staff at baseline. Current smokers or current alcohol drinkers were patients who smoked more than one cigarette per day or drank alcohol more than once per week continuously during past six months, and patients who had quit smoking or drinking at diagnosis were former smokers or former drinkers. Body mass index (BMI) was calculated based on height $(\mathrm{m})$ and weight $(\mathrm{kg})$ at diagnosis. In addition, education level, age at diagnosis, sex, residence and family history of primary liver cancer were also collected.

We retrospectively extracted examination, diagnostic and treatment data from the SYSUCC electronic medical records. Laboratory data at diagnosis included albumin (ALB), total bilirubin (TBIL), alkaline phosphatase (ALP), $\gamma$-glutaryl-transferase (GGT), aspartate aminotransferase (AST), alanine aminotransferase (ALT), prothrombin time (PT), alpha fetoprotein (AFP), C-reactive protein (CRP), hepatitis $B$ surface antigen ( $\mathrm{HBsAg}$ ) and hepatitis $\mathrm{C}$ virus antibody (Anti-HCV). A liver damage level was computed based on six liver function tests (ALB, TBIL, ALP, GGT, AST and ALT), cutoffs were described previously [22], the level was presented as 1 (no liver injury), 2 (possible minor injury), and $\geq 3$ (possible injury) in this study. Characteristics of HCC tumor and the non-tumoral liver were reviewed through imaging or pathology data at diagnosis, then Child-Pugh class [25] and Barcelona Clinic Liver Cancer (BCLC) stage [25] were determined. Treatments mainly included liver resection, intervention, radiofrequency ablation and others. In addition, presence of chronic diseases at diagnosis which may affect the 
prognosis of HCC were also collected, including hypertension, diabetes mellitus, fatty liver and cirrhosis.

\section{Statistical analysis}

The statistical analyses were computed in SPSS version 20.0 for WINDOWS (SPSS Inc., Chicago, IL, USA). All $P$-values were based on two-sided tests, and $P<0.05$ was considered statistically significant.

The serum levels of choline and betaine at diagnosis were mostly rescaled to sex-specific tertiles ( $T$ ) for analysis, except for the stratified analyses. Differences in basic characteristics across sex-specific tertiles of serum choline and betaine were compared by one-way analysis of variance (ANOVA), Kruskal-Wallis tests or Pearson's Chi-Squared tests, where appropriate. Multivariate Cox proportional hazards models were used to examine the relationship between serum levels of choline and betaine and HCC survival outcomes. Hazard ratios (HRs) and 95\% confidence interval (CI) were calculated with the first tertiles as the reference. Before performing multivariate analysis, covariates were preliminarily screened using the nonparametric logrank tests $(P$-values for log-rank test are presented in Additional file 1: Supplementary Table 1), and those with $P$ values $>0.05$ were firstly excluded. Then the multicollinearity was evaluated using the variation inflation factor and tolerance. Presence of fatty liver, presence of cirrhosis and Child-Pugh class were further excluded, due to the multicollinearity with the baseline liver damage level or BCLC stage. Particularly, age at diagnosis and sex (log-rank test $P$ values $>0.05$ ) were retained while residence (log-rank test $P$-value $<0.05)$ was excluded, since age and sex are wellestablished HCC prognostic factors. Finally, in model 1, nonclinical covariates such as age at diagnosis, sex, education level, BMI and smoking status were selected to adjust. In model 2, significant clinical covariates were further adjusted, including AFP level ( $\geq 400$ or $<400 \mathrm{ng} / \mathrm{L}$ ), CRP level $(\geq 3.0$ or $<3 \mathrm{mg} / \mathrm{L}$ ), baseline liver damage level, BCLC stage and treatments. Linear trends $\left(P_{\text {-trend }}\right)$ were evaluated by entering sex-specific tertiles of serum choline and betaine as continuous variables in the corresponding models.

Stratified analyses were conducted to evaluate whether the relationships between serum choline and betaine and HCC survival would be modified by potential prognosis factors for HCC, including sex, age at diagnosis, BMI, smoking status, alcohol drinking status, serum folate level, AFP level, CRP level, baseline liver damage level, presence of fatty liver, presence of cirrhosis, BCLC stage and treatments. Interactions were estimated by including the multiplicative interaction terms in the multivariate models.

\section{Results}

\section{Patient characteristics}

Basic characteristics of the 866 HCC patients by sex are presented in Additional file 2 (Supplementary Table 2), and by sex-specific tertiles of serum choline and betaine are shown in Table 1 . Of the 866 patients, there were 767 $(88.6 \%)$ men. The mean $( \pm$ S.D. $)$ age was $52.3 \pm 11.8$ years. The median (IQR) levels of serum choline and betaine were 11.72 (9.52-14.92) $\mu \mathrm{mol} / \mathrm{L}$ and 64.48 (52.44-77.65) $\mu \mathrm{mol} / \mathrm{L}$, respectively. Compared to subjects in the bottom sex-specific tertiles of serum choline, those in the top tertiles were older, less likely to be current smokers, more likely to be former smokers and were less been diagnosed as BCLC C stage. Compared to patients in the bottom sex-specific tertiles of serum betaine, those in the top tertiles were more living in rural, more infected by HBV or HCV and less suffered from fatty liver.

\section{Associations between serum choline and betaine with HCC survival outcomes}

The median survival of HCC patients was 467 days (IQR: 227-835 days). During the follow-up, 291 (33.6\%) deaths were documented, $270(92.8 \%)$ of which were attributable to liver cancer. Both liver cancer-specific and overall mortality decreased with sex-specific tertiles of serum choline levels after adjusting for nonclinical factors in model 1. The associations became even stronger after further adjustment for traditional HCC prognostic factors in model 2, HRs (95\% CIs) of liver cancer-specific and overall mortality (T3 vs T1) in model 2 were 0.69 (0.51$0.94)$ and $0.73(0.54-0.99)$, respectively. Both $P_{\text {-trend }}$ values were $<0.05$. However, no significant associations were found between serum betaine levels and either LCSS or OS in the fully-adjusted model 2 (all $P_{\text {-trend }}$ values were $>0.05$ ). Data are presented in Table 2.

\section{Stratified analyses}

Stratified analyses of serum choline and betaine levels by selected factors are shown in Tables 3 and 4. No statistically significant multiplicative effect modifications were found across most of the aforementioned strata factors, either for LCSS or OS (all $P_{\text {-interaction }}>0.05$ ). However, we observed significant multiplicative effect modifications by CRP level for serum choline. Compared to the first tertile of serum choline, patients in the third tertile had better survival in CRP $\geq 3.0 \mathrm{mg} / \mathrm{L}$ strata (HRs $(95 \%$ CIs) of LCSS and OS (T3 vs T1) were $0.60(0.41-0.88)$ and $0.63(0.44-0.91), P_{\text {-interaction }}=0.010$ and 0.005 , respectively.) but not in CRP $<3.0 \mathrm{mg} / \mathrm{L}$ strata.

\section{Discussion}

To our best knowledge, this is the first study to examine serum choline and betaine in relation to HCC survival in a large cohort. We observed that higher serum choline levels at diagnosis were associated with better HCC survival, especially in those with systemic inflammation status (CRP level $\geq 3.0 \mathrm{mg} / \mathrm{L}$ ), whereas serum betaine 
Table 1 Patient characteristics at diagnosis by sex-specific tertiles of serum choline/betaine levels

\begin{tabular}{|c|c|c|c|c|c|c|c|c|}
\hline & \multicolumn{4}{|c|}{ Serum choline } & \multicolumn{4}{|c|}{ Serum betaine } \\
\hline & $\overline{\mathrm{T} 1}$ & $\mathrm{~T} 2$ & T3 & $\boldsymbol{P}$-value ${ }^{\mathbf{b}}$ & $\overline{\mathrm{T} 1}$ & $\mathrm{~T} 2$ & T3 & $\boldsymbol{P}$-value ${ }^{\mathbf{b}}$ \\
\hline Age at diagnosis ${ }^{a}$, years & $50.2 \pm 11.9$ & $52.8 \pm 11.5$ & $53.8 \pm 11.7$ & 0.00 & $51.6 \pm 11.7$ & $52.1 \pm 12.2$ & $51.6 \pm 11.7$ & 0.37 \\
\hline Men, $n(\%)$ & $257(88.6)$ & $257(88.9)$ & $256(89.2)$ & 0.98 & $256(88.9)$ & $258(89.0)$ & $256(88.9)$ & 1.00 \\
\hline BMI at diagnosis ${ }^{a}, \mathrm{~kg} / \mathrm{m}^{2}$ & $22.5 \pm 3.3$ & $22.8 \pm 3.2$ & $22.7 \pm 3.3$ & 0.34 & $22.5 \pm 3.0$ & $22.9 \pm 3.6$ & $22.5 \pm 3.0$ & 0.68 \\
\hline Education level, $n$ (\%) & & & & 0.37 & & & & 0.45 \\
\hline Primary school or below & $56(19.4)$ & $63(21.9)$ & $52(18.1)$ & & $62(21.7)$ & $48(16.6)$ & $61(21.2)$ & \\
\hline Secondary \& High school & $168(58.3)$ & $177(61.5)$ & $183(63.8)$ & & $175(61.2)$ & $184(63.7)$ & $169(58.7)$ & \\
\hline College or above & $64(22.2)$ & $48(16.7)$ & $52(18.1)$ & & $49(17.1)$ & $57(19.7)$ & $58(20.1)$ & \\
\hline Residence, $n$ (\%) & & & & 0.60 & & & & 0.03 \\
\hline Urban & $195(67.2)$ & $205(70.9)$ & $195(67.9)$ & & $215(74.7)$ & $192(66.2)$ & $188(65.3)$ & \\
\hline Rural & $95(32.8)$ & $84(29.1)$ & $92(32.1)$ & & $73(25.3)$ & $98(33.8)$ & $100(34.7)$ & \\
\hline Smoking status, $\boldsymbol{n}(\%)$ & & & & 0.00 & & & & 0.06 \\
\hline Current & $101(34.8)$ & $90(31.1)$ & $74(25.8)$ & & $106(36.8)$ & $81(27.9)$ & $78(27.1)$ & \\
\hline former & $55(19.0)$ & $86(29.8)$ & $100(34.8)$ & & $71(24.7)$ & $80(27.6)$ & $90(31.2)$ & \\
\hline Never & $134(46.2)$ & $113(39.1)$ & $113(39.4)$ & & $111(38.5)$ & $129(44.5)$ & $120(41.7)$ & \\
\hline Alcohol drinking status, $\boldsymbol{n}$ (\%) & & & & 0.57 & & & & 0.73 \\
\hline Current & $83(28.6)$ & $75(26.0)$ & $79(27.5)$ & & $75(26.0)$ & $86(29.7)$ & $76(26.4)$ & \\
\hline former & $39(13.4)$ & $37(12.8)$ & $48(16.7)$ & & $41(14.2)$ & $37(12.8)$ & $46(16.0)$ & \\
\hline Never & $168(57.9)$ & $177(61.2)$ & $160(55.7)$ & & $172(59.7)$ & $167(57.6)$ & $166(57.6)$ & \\
\hline With family history of PLC, $n(\%)$ & $46(15.9)$ & $33(11.4)$ & $33(11.5)$ & 0.19 & $37(12.8)$ & $39(13.4)$ & $36(12.5)$ & 0.94 \\
\hline HBV or HCV infected, $n(\%)$ & $269(92.8)$ & $263(91.0)$ & $257(89.5)$ & 0.40 & $254(88.2)$ & $261(90.0)$ & $274(95.1)$ & 0.01 \\
\hline AFP $\geq 400$ ng/L, $n(\%)$ & $112(38.9)$ & $130(45.0)$ & $116(40.4)$ & 0.30 & $124(43.2)$ & $124(42.9)$ & $110(38.2)$ & 0.39 \\
\hline $\mathrm{CRP} \geq 3.0 \mathrm{mg} / \mathrm{L}, n(\%)$ & $148(51.6)$ & $139(48.3)$ & $149(52.1)$ & 0.61 & $138(48.1)$ & $137(47.6)$ & $161(56.3)$ & 0.06 \\
\hline \multicolumn{9}{|l|}{ Presence of chronic diseases, $n(\%)$} \\
\hline Hypertension & $28(9.7)$ & $44(15.2)$ & $39(13.6)$ & 0.12 & $34(11.7)$ & $33(11.5)$ & $111(12.8)$ & 0.31 \\
\hline Diabetes mellitus & $19(6.6)$ & $21(7.3)$ & $29(10.1)$ & 0.25 & $30(10.4)$ & $22(7.6)$ & $17(5.9)$ & 0.13 \\
\hline Fatty liver & $44(15.2)$ & $50(17.3)$ & $49(17.1)$ & 0.75 & $64(22.2)$ & $47(16.2)$ & $32(11.1)$ & 0.00 \\
\hline Cirrhosis & $176(60.7)$ & $186(64.4)$ & $182(63.4)$ & 0.64 & $175(60.8)$ & $176(60.7)$ & $193(67.0)$ & 0.20 \\
\hline Baseline liver damage level, $n$ (\%) & & & & 0.87 & & & & 0.16 \\
\hline 1 & $58(20.0)$ & $62(21.5)$ & $52(18.1)$ & & $60(20.8)$ & $58(20.0)$ & $54(18.8)$ & \\
\hline 2 & $109(37.6)$ & $109(37.7)$ & $116(40.4)$ & & $95(33.0)$ & $123(42.4)$ & $116(40.3)$ & \\
\hline 3 & $123(42.4)$ & $118(40.8)$ & $119(41.5)$ & & $133(46.2)$ & $109(37.6)$ & $118(41.0)$ & \\
\hline Child-Pugh class, $n$ (\%) & & & & 0.45 & & & & 0.16 \\
\hline A & $283(97.6)$ & $286(99.0)$ & $282(98.3)$ & & $286(99.3)$ & $285(98.3)$ & $280(97.2)$ & \\
\hline B & $7(2.4)$ & $3(1.0)$ & $5(1.7)$ & & $2(0.7)$ & $5(1.7)$ & $8(2.8)$ & \\
\hline BCLC stage, $n(\%)$ & & & & 0.01 & & & & 0.95 \\
\hline 0 & $23(7.9)$ & $31(10.7)$ & $28(9.8)$ & & $25(8.7)$ & $28(9.7)$ & $29(10.1)$ & \\
\hline A & $85(29.3)$ & $80(27.7)$ & $101(35.2)$ & & $87(30.2)$ & $95(32.8)$ & $84(29.2)$ & \\
\hline B & $20(6.9)$ & $41(14.2)$ & $34(11.8)$ & & $33(11.5)$ & $32(11.0)$ & $30(10.4)$ & \\
\hline C & $162(55.9)$ & $137(47.4)$ & $124(43.2)$ & & $143(49.7)$ & $135(46.6)$ & $145(50.3)$ & \\
\hline Treatment, $n(\%)$ & & & & 0.61 & & & & 0.18 \\
\hline Liver resection & $132(45.5)$ & $124(42.9)$ & $127(44.3)$ & & $133(46.2)$ & $134(46.2)$ & $116(40.3)$ & \\
\hline Radiofrequency ablation & $31(10.7)$ & $28(9.7)$ & $26(9.1)$ & & $21(7.3)$ & $29(10.0)$ & $35(12.2)$ & \\
\hline Intervention & 109 (37.6) & $127(43.9)$ & $121(42.2)$ & & $121(42.0)$ & 109 (37.6) & $127(44.1)$ & \\
\hline
\end{tabular}


Table 1 Patient characteristics at diagnosis by sex-specific tertiles of serum choline/betaine levels (Continued)

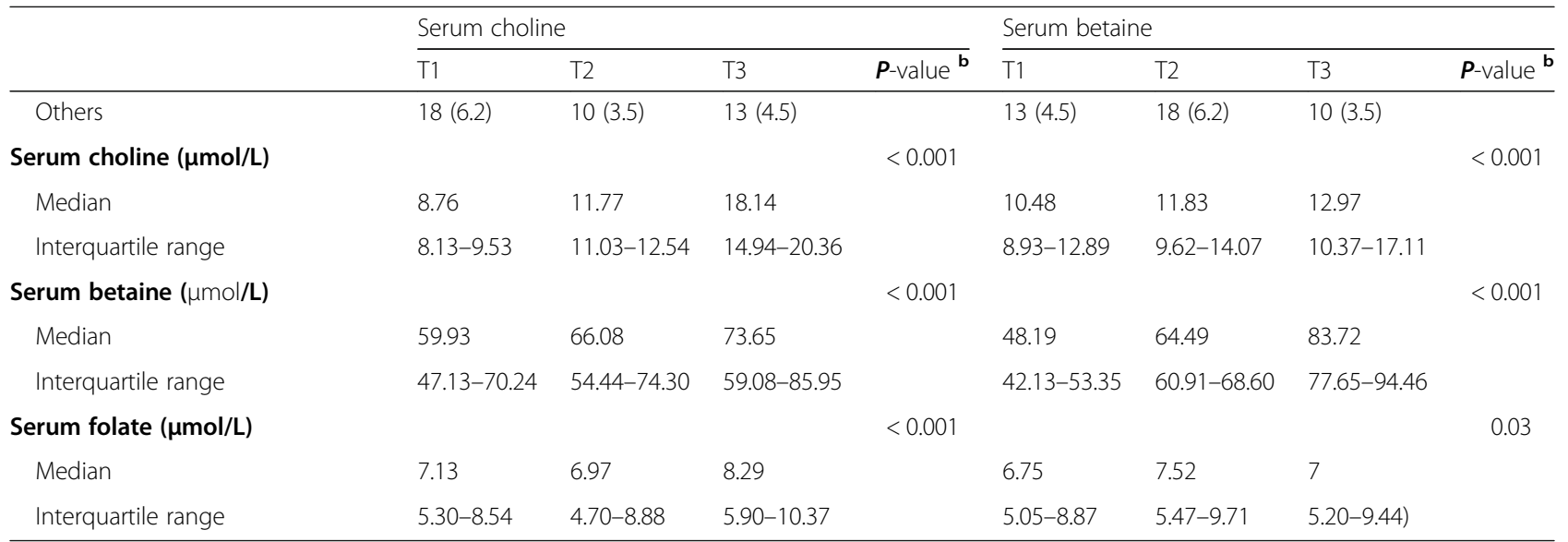

${ }^{a}$ values are expressed as mean \pm S.D.

b $P$-values were calculated by one-way analysis of variance (ANOVA), Kruskal-Wallis tests and Pearson's Chi-Squared tests, where appropriate

had no statistically significant associations with HCC survival outcomes.

Given its critically protective role for liver and other essential physiological functions in the body, it is not surprising that serum choline demonstrated favorable associations with HCC survival. First, choline is an essential nutrient for maintaining liver function. Liver is among the first organs to accumulate choline absorbed from the intestine and responsible for most of choline metabolism [26]. Experimental studies have proved that

Table 2 Survival outcomes by sex-specific tertiles of serum choline and betaine levels

\begin{tabular}{|c|c|c|c|c|}
\hline & $\mathrm{T1}$ & $\mathrm{T} 2$ & T3 & $P_{\text {-trend }}$ \\
\hline \multicolumn{5}{|l|}{ Liver cancer-specific survival } \\
\hline \multicolumn{5}{|l|}{ Serum choline } \\
\hline Deaths / Total cases & 87 / 290 & $84 / 289$ & 99 / 287 & \\
\hline Person-days at risk & 122,905 & 143,328 & 202,856 & \\
\hline Model 1-adjusted HR $(95 \% \mathrm{Cl})^{\text {a }}$ & 1.00 & $0.83(0.61-1.13)$ & $0.73(0.55-0.99)$ & 0.04 \\
\hline Model 2-adjusted HR $(95 \% \mathrm{Cl})^{\mathrm{b}}$ & 1.00 & $0.84(0.62-1.15)$ & $0.69(0.51-0.94)$ & 0.02 \\
\hline \multicolumn{5}{|l|}{ Serum betaine } \\
\hline Deaths / Total cases & $90 / 288$ & $94 / 290$ & $86 / 288$ & \\
\hline Person-days at risk & 149,722 & 156,715 & 162,652 & \\
\hline Model 1-adjusted HR $(95 \% \mathrm{Cl})^{\text {a }}$ & 1.00 & $0.94(0.70-1.26)$ & $0.85(0.63-1.15)$ & 0.29 \\
\hline Model 2-adjusted HR $(95 \% \mathrm{CI})^{\text {b }}$ & 1.00 & $0.97(0.72-1.31)$ & $0.84(0.62-1.13)$ & 0.25 \\
\hline \multicolumn{5}{|l|}{ Overall survival } \\
\hline \multicolumn{5}{|l|}{ Serum choline } \\
\hline Deaths / Total cases & $90 / 290$ & $91 / 289$ & $110 / 287$ & \\
\hline Person-days at risk & 122,905 & 143,328 & 202,856 & \\
\hline Model 1-adjusted HR $(95 \% \mathrm{CI})^{\text {a }}$ & 1.00 & $0.85(0.63-1.14)$ & $0.78(0.58-1.05)$ & 0.11 \\
\hline Model 2-adjusted HR $(95 \% \mathrm{Cl})^{\mathrm{b}}$ & 1.00 & $0.85(0.62-1.15)$ & $0.73(0.54-0.99)$ & 0.04 \\
\hline \multicolumn{5}{|l|}{ Serum betaine } \\
\hline Deaths / Total cases & $97 / 288$ & $99 / 290$ & $95 / 288$ & \\
\hline Person-days at risk & 149,722 & 156,715 & 162,652 & \\
\hline Model 1-adjusted HR $(95 \% \mathrm{Cl})^{\text {a }}$ & 1.00 & $0.93(0.70-1.24)$ & $0.88(0.66-1.17)$ & 0.39 \\
\hline Model 2-adjusted HR $(95 \% \mathrm{Cl})^{\mathrm{b}}$ & 1.00 & $0.95(0.72-1.27)$ & $0.84(0.63-1.13)$ & 0.25 \\
\hline
\end{tabular}

\footnotetext{
${ }^{a}$ Covariates adjusted in Model 1: age, sex, BMI, education level, smoking status
}

${ }^{\mathrm{b}}$ Covariates adjusted in Model 2: covariates adjusted in Model 1, additionally adjusted for baseline liver damage level, BCLC stage, treatment, AFP level and CRP level 
Table 3 HR (95\% Cl) of stratified analysis across tertiles of serum choline levels

\begin{tabular}{|c|c|c|c|c|c|c|c|c|c|c|}
\hline & \multicolumn{5}{|c|}{ Liver cancer-specific survival } & \multicolumn{4}{|c|}{ Overall survival } & \multirow[b]{2}{*}{$P_{\text {-interaction }}$} \\
\hline & $\mathrm{T1}$ & $\mathrm{T} 2$ & T3 & $P_{\text {-trend }}$ & $P_{\text {-interaction }}$ & $\mathrm{T1}$ & $\mathrm{T} 2$ & T3 & $P_{\text {-trend }}$ & \\
\hline $\operatorname{Sex}^{a, b}$ & & & & & 0.511 & & & & & 0.781 \\
\hline Men & 1.00 & $0.77(0.56-1.07)$ & $0.66(0.48-0.92)$ & 0.013 & & 1.00 & $0.80(0.58-1.10)$ & $0.71(0.52-0.98)$ & 0.037 & \\
\hline Women & 1.00 & $1.21(0.41-3.52)$ & $1.59(0.57-4.44)$ & 0.360 & & 1.00 & $1.15(0.42-3.14)$ & $1.33(0.50-3.54)$ & 0.567 & \\
\hline Age ${ }^{a, b}$, years & & & & & 0.838 & & & & & 0.801 \\
\hline$<60$ & 1.00 & $1.03(0.72-1.48)$ & $0.69(0.48-0.99)$ & 0.031 & & 1.00 & $1.07(0.75-1.52)$ & $0.74(0.52-1.06)$ & 0.070 & \\
\hline$\geq 60$ & 1.00 & $0.80(0.42-1.51)$ & $0.63(0.34-1.17)$ & 0.142 & & 1.00 & $0.83(0.45-1.53)$ & $0.65(0.36-1.18)$ & 0.155 & \\
\hline $\mathrm{BMI}^{\mathrm{a}}, \mathrm{b}, \mathrm{kg} / \mathrm{m}^{2}$ & & & & & 0.357 & & & & & 0.436 \\
\hline$<25.0$ & 1.00 & $0.90(0.64-1.27)$ & $0.74(0.53-1.04)$ & 0.079 & & 1.00 & $0.88(0.62-1.23)$ & $0.77(0.55-1.07)$ & 0.117 & \\
\hline$\geq 25.0$ & 1.00 & $1.15(0.54-2.44)$ & $0.47(0.20-1.10)$ & 0.118 & & 1.00 & $1.03(0.50-2.12)$ & $0.61(0.28-1.32)$ & 0.185 & \\
\hline Smoking status ${ }^{a, b}$ & & & & & 0.820 & & & & & 0.756 \\
\hline Smoker & 1.00 & $0.58(0.39-0.85)$ & $0.57(0.39-0.83)$ & 0.010 & & 1.00 & $0.61(0.42-0.89)$ & $0.61(0.42-0.88)$ & 0.013 & \\
\hline Non-smoker & 1.00 & $1.17(0.69-1.98)$ & $0.85(0.50-1.42)$ & 0.481 & & 1.00 & $1.19(0.71-2.01)$ & $0.91(0.55-1.51)$ & 0.669 & \\
\hline Drinking status ${ }^{a, b}$ & & & & & 0.388 & & & & & 0.399 \\
\hline Drinker & 1.00 & $0.67(0.41-1.07)$ & $0.78(0.50-1.22)$ & 0.342 & & 1.00 & $0.71(0.44-1.13)$ & $0.75(0.48-1.16)$ & 0.237 & \\
\hline Non-Drinker & 1.00 & $1.23(0.82-1.86)$ & $0.72(0.47-1.12)$ & 0.077 & & 1.00 & $1.16(0.77-1.74)$ & $0.74(0.49-1.13)$ & 0.135 & \\
\hline AFP level ${ }^{a}, b, n g / m L$ & & & & & 0.160 & & & & & 0.566 \\
\hline$\geq 400$ & 1.00 & $0.85(0.56-1.30)$ & $0.80(0.52-1.22)$ & 0.300 & & 1.00 & $0.86(0.57-1.29)$ & $0.83(0.56-1.25)$ & 0.385 & \\
\hline$<400$ & 1.00 & $0.87(0.55-1.37)$ & $0.68(0.43-1.06)$ & 0.085 & & 1.00 & $0.91(0.58-1.42)$ & $0.73(0.48-1.13)$ & 0.155 & \\
\hline CRP level ${ }^{a, b}, \mathrm{mg} / \mathrm{L}$ & & & & & 0.010 & & & & & 0.005 \\
\hline$\geq 3.0$ & 1.00 & $0.91(0.64-1.29)$ & $0.60(0.41-0.88)$ & 0.008 & & 1.00 & $0.92(0.65-1.29)$ & $0.63(0.44-0.91)$ & 0.013 & \\
\hline$<3.0$ & 1.00 & $1.34(0.70-2.56)$ & $1.37(0.74-2.51)$ & 0.353 & & 1.00 & $1.34(0.71-2.54)$ & $1.47(0.81-2.67)$ & 0.224 & \\
\hline Serum folate level ${ }^{a, b}$ & & & & & 0.782 & & & & & 0.946 \\
\hline High & 1.00 & $1.07(0.67-1.70)$ & $0.79(0.50-1.26)$ & 0.279 & & 1.00 & $1.17(0.74-1.84)$ & $0.91(0.58-1.43)$ & 0.583 & \\
\hline Low & 1.00 & $0.96(0.62-1.47)$ & $0.77(0.50-1.17)$ & 0.207 & & 1.00 & $0.94(0.62-1.42)$ & $0.76(0.50-1.14)$ & 0.170 & \\
\hline Liver damage level ${ }^{a, b}$ & & & & & 0.520 & & & & & 0.602 \\
\hline 1 & 1.00 & $1.39(0.34-5.74)$ & $1.54(0.40-6.00)$ & 0.547 & & 1.00 & $1.85(0.52-6.62)$ & $1.16(0.34-3.97)$ & 0.935 & \\
\hline 2 & 1.00 & $1.06(0.63-1.78)$ & $0.58(0.33-1.01)$ & 0.045 & & 1.00 & $1.06(0.63-1.78)$ & $0.65(0.38-1.13)$ & 0.114 & \\
\hline$\geq 3$ & 1.00 & $0.82(0.55-1.22)$ & $0.72(0.48-1.08)$ & 0.115 & & 1.00 & $0.86(0.58-1.26)$ & $0.78(0.53-1.14)$ & 0.206 & \\
\hline Presence of fatty liver ${ }^{a, b}$ & & & & & 0.385 & & & & & 0.842 \\
\hline Yes & 1.00 & $1.08(0.38-3.07)$ & $0.94(0.32-2.78)$ & 0.902 & & 1.00 & $1.05(0.40-2.76)$ & $1.17(0.45-3.03)$ & 0.741 & \\
\hline No & 1.00 & $0.94(0.68-1.30)$ & $0.69(0.50-0.97)$ & 0.026 & & 1.00 & $0.96(0.70-1.32)$ & $0.72(0.52-0.99)$ & 0.035 & \\
\hline Presence of cirrhosis ${ }^{a, b}$ & & & & & 0.518 & & & & & 0.170 \\
\hline Yes & 1.00 & $0.93(0.60-1.43)$ & $0.83(0.54-1.27)$ & 0.380 & & 1.00 & $0.93(0.61-1.42)$ & $0.90(0.60-1.35)$ & 0.602 & \\
\hline No & 1.00 & $0.98(0.63-1.52)$ & $0.63(0.39-1.00)$ & 0.048 & & 1.00 & $1.01(0.66-1.56)$ & $0.62(0.39-0.98)$ & 0.039 & \\
\hline BCLC stage ${ }^{a, b}$ & & & & & 0.774 & & & & & 0.502 \\
\hline O/A & 1.00 & $0.80(0.34-1.90)$ & $0.72(0.32-1.62)$ & 0.440 & & 1.00 & $0.91(0.40-2.11)$ & $0.78(0.35-1.73)$ & 0.515 & \\
\hline$B / C$ & 1.00 & $0.89(0.64-1.23)$ & $0.64(0.46-0.90)$ & 0.008 & & 1.00 & $0.88(0.63-1.21)$ & $0.67(0.48-0.93)$ & 0.014 & \\
\hline Treatment ${ }^{\mathrm{a}, \mathrm{b}}$ & & & & & 0.333 & & & & & 0.480 \\
\hline Liver resection & 1.00 & $1.29(0.69-2.41)$ & $0.81(0.42-1.56)$ & 0.536 & & 1.00 & $1.16(0.63-2.14)$ & $0.87(0.46-1.62)$ & 0.664 & \\
\hline Intervention & 1.00 & $0.75(0.51-1.10)$ & $0.60(0.41-0.86)$ & 0.006 & & 1.00 & $0.80(0.55-1.17)$ & $0.64(0.45-0.92)$ & 0.015 & \\
\hline Others & 1.00 & $1.26(0.52-3.07)$ & $0.95(0.37-2.42)$ & 0.908 & & 1.00 & $1.21(0.49-2.94)$ & $1.00(0.40-2.50)$ & 0.985 & \\
\hline
\end{tabular}

\footnotetext{
${ }^{a}$ Covariates adjusted for age, sex, BMI, education level, smoking status, baseline liver damage level, BCLC stage, treatment, AFP level and CRP level
}

${ }^{\mathrm{b}}$ Stratified factors were not included in the corresponding models 
Table $4 \mathrm{HR}(95 \% \mathrm{Cl})$ of stratified analysis across tertiles of serum betaine levels

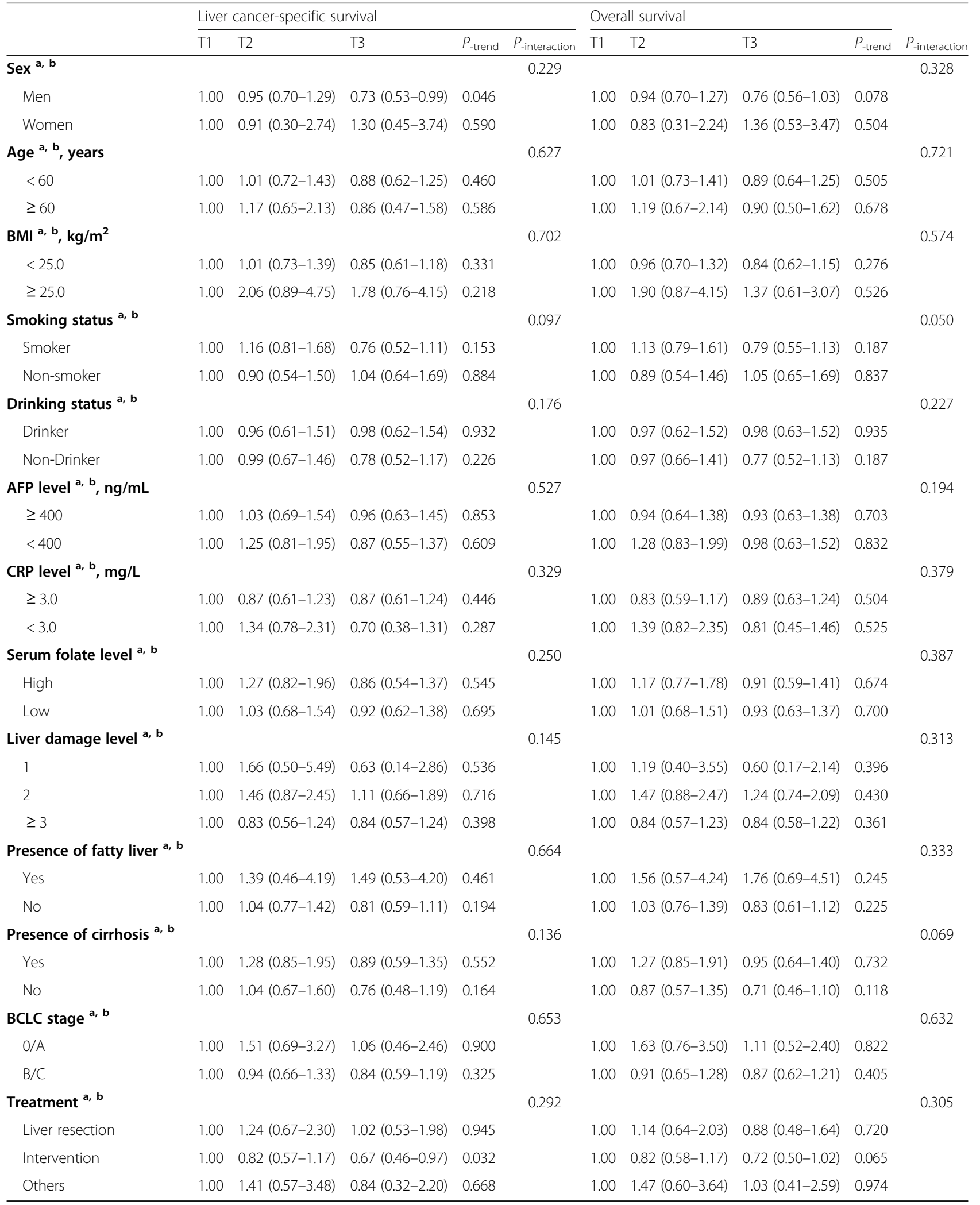

\footnotetext{
${ }^{a}$ Covariates adjusted for age, sex, BMl, education level, smoking status, baseline liver damage level, BCLC stage, treatment, AFP level and CRP level
}

${ }^{\mathrm{b}}$ Stratified factors were not included in the corresponding models 
choline deficiency might cause varying degrees of liver diseases ranging from dyslipidemia to liver cancer [26], whereas choline or betaine supplementation could ameliorate liver damage $[15,16]$. Second, though choline is essential for maintaining normal liver function, its functions are more than this. It is indispensable for normal function of all cells, including the structural integrity of cell membranes and neurotransmission [27]. Thus, adequate choline can improve general health of HCC patients so as to further improve HCC survival outcomes. Third, choline is a major source of methyl group via oxidation to betaine, their important roles in methylation reactions are critical for DNA and histone methylation homeostasis. Jiang et al. [17] used the human hepatic HepG2 cell to examine the effect of choline supplementation on DNA methylation, they found that choline supplementation increased both global DNA methylation and DNA methyltransferase expression, suggesting that choline could improve prognosis of HCC. However, in the present study, the favorable associations were only observed between serum choline and HCC survival outcomes, and no statistically significant associations between serum betaine and HCC survival outcomes were found. These contradictory results implied that the protective effect of choline on HCC survival may not through the methylation metabolic pathway via oxidation to betaine. As a methyl donor, betaine participates in the re-methylation of homocysteine to methionine via the enzyme betaine homocysteine methyltransferase (BHMT) [26]. However, this key enzyme is greatly reduced in HCC patients [28], and downregulation of BHMT in HCC associates with poor prognosis [29]. Besides, after treating the Hepa 1-6 (derived cells from a mouse HCC model) and E47/C34 cell lines (clones of the HepG2 cell line) with exogenous S-adenosylmethionine (SAM) and betaine, results showed that SAM decreased the number of Hepa 1-6 and E47/C34 cells, and increased the number of dead cells in vitro, while betaine had no significant effect either on the number of surviving cells or dead cells [30]. Lastly, in the stratified analysis, we observed significant multiplicative effect modifications by CRP level, the favorable association between serum choline and HCC survival was only found in CRP $\geq 3.0 \mathrm{mg} / \mathrm{L}$ strata, but not in CRP $<3.0$ $\mathrm{mg} / \mathrm{L}$ strata. As a marker of systemic inflammation status, CRP has been identified as a useful prognostic factor for HCC [31]. Our results implied that elevating serum choline may have more beneficial effects on HCC patients with systemic inflammation status.

Several strengths of this study should be noted. This is the first large prospective cohort study to investigate the associations of serum choline with HCC survival in a Chinese population, whose dietary choline intake were seriously insufficient $[20,21]$, thus the value of this study is significant and our findings may have impact for the design of future clinical trials. We only enrolled newly diagnosed HCC patients and collected blood samples within 1 months of diagnosis, to eliminate potential confounding by receiving any anticancer treatments. Furthermore, we collected very detailed information of the HCC patients, including both general and clinical prognostic factors.

However, some potential limitations should also be considered. We failed to collect the information of dietary habits changes and disease progression during the followup, and we had only one measurement of serum choline and betaine at diagnosis, due to the high mortality and poor prognosis of HCC, another time-consuming face-toface interview and blood collection was extremely difficult, thus we cannot know the circulating choline and betaine changes after diagnosis. In addition, it should be noted that our study is a preliminary epidemiological research, although to some extent, our finding had opened up new prospect in understanding the beneficial role of choline on HCC survival, we cannot explain the exact mechanisms restricted by the observational study design, further investigations such as randomized clinical trials are warranted to confirm these findings.

\section{Conclusions}

In conclusion, the key findings from this large prospective cohort study revealed that higher serum choline levels at diagnosis were associated with better HCC survival outcomes, independently from representative nonclinical and clinical prognostic factors, especially in HCC patients with systemic inflammation status. Further investigations such as randomized clinical trials are warranted to confirm these findings.

\section{Supplementary information}

Supplementary information accompanies this paper at https://doi.org/10. 1186/s12986-020-00445-z.

Additional file $\mathbf{1}$ Table $\mathbf{S 1}$. Screening procedure of variables by nonparametric log-rank test.

Additional file $\mathbf{2}$ Table $\mathbf{5 2}$. Baseline characteristics of female and male HCC patients.

\section{Abbreviations \\ HCC: Hepatocellular carcinoma; GLCC: Guangdong Liver Cancer Cohort; LCSS: Liver cancer-specific survival; OS: Overall survival; HRs: Hazard ratios; Cls: Confidence intervals; SYSUCC: Sun Yat-sen University Cancer Center; PLC: Primary liver cancer; T1: First tertile; T2: Second tertile; T3: Third tertile; BMI: Body mass index; HBV: Hepatitis B virus; HCV: Hepatitis C virus; AFP: Alpha fetoprotein; CRP: C-reactive protein; BCLC: Barcelona Clinic Liver Cancer; ALB: Albumin; TBIL: Total bilirubin; ALP: Alkaline phosphatase; GGT: Y- glutaryl-transferase; AST: Aspartate aminotransferase; ALT: Alanine aminotransferase; PT: Prothrombin time; SAM: S-adenosylmethionine; BHMT: Betaine homocysteine methyltransferase}

\section{Acknowledgements}

We thank all the HCC patients and research stuff for their contributions to this study. 


\section{Authors' contributions}

ZYL performed the study design, data analysis and drafted the manuscript. HLZ, YJZ and APF administrated the project. ZYL, DY, DMZ, GCL and XYT collected the data and blood samples, and conducted the choline and betaine analysis. All authors read and approved the final manuscript.

\section{Funding}

This research was funded by the National Natural Science Foundation of China, grant number 81973016 and 81803219 , and the Natural Science Foundation of Guangdong Province, China, grant number 2018A030310335.

\section{Availability of data and materials}

The datasets generated and/or analyzed during the current study are not publicly available due to privacy protection of the participants but are available from the corresponding author on reasonable request.

\section{Ethics approval and consent to participate}

The Ethics Committee of the School of Public Health at Sun Yat-sen University approved the protocol of this study. Written informed consent was obtained from all participants. The study was performed in accordance with the Declaration of Helsinki.

\section{Consent for publication}

Not applicable.

\section{Competing interests}

The authors declare that they have no competing interests.

\section{Author details}

${ }^{1}$ Guangdong Provincial Key Laboratory of Food, Nutrition and Health, School of Public Health, Sun Yat-sen University, Guangzhou 510080, People's Republic of China. ${ }^{2}$ Department of Hepatobiliary Surgery, Sun Yat-sen University Cancer Center, Guangzhou 510060, People's Republic of China.

Received: 26 December 2019 Accepted: 21 March 2020

Published online: 30 March 2020

\section{References}

1. Bray F, Ferlay J, Soerjomataram I, Siegel RL, Torre LA, Jemal A. Global cancer statistics 2018: GLOBOCAN estimates of incidence and mortality worldwide for 36 cancers in 185 countries. CA Cancer J Clin. 2018;68:394-424.

2. Youn J, Cho E, Lee JE. Association of choline and betaine levels with cancer incidence and survival: A meta-analysis. Clin Nutr. 2019:38(1):100-9.

3. World cancer research fund/American Institute for Cancer Research. Continuous Update Project Expert Report 2018. Diet, nutrition, physical activity and liver cancer. Available at https://www.wcrforg/dietandcancer.

4. Harding JJ, Khalil DN, Abou-Alfa GK. Biomarkers: what role do they play (if any) for diagnosis, prognosis and tumor response prediction for hepatocellular carcinoma? Dig Dis Sci. 2019;64(4):918-27.

5. Hollenbeck CB. An introduction to the nutrition and metabolism of choline. Cent Nerv Syst Agents Med Chem. 2012;12:100-13.

6. Zeisel SH. Choline: critical role during fetal development and dietary requirements in adults. Annu Rev Nutr. 2006;26:229-50

7. Richman EL, Kenfield SA, Stampfer MJ, Giovannucci EL, Zeisel SH, Willett WC Chan JM. Choline intake and risk of lethal prostate cancer: incidence and survival. Am J Clin Nutr. 2012:96:855-63.

8. Dixon SC, Ibiebele TI, Protani MM, Beesley J, deFazio A, Crandon AJ, Gard GB, Rome RM, Webb PM, Nagle CM, Australian Ovarian Cancer Study G. Dietary folate and related micronutrients, folate-metabolising genes, and ovarian cancer survival. Gynecol Oncol. 2014;132:566-72.

9. Galvan-Portillo MV, Onate-Ocana LF, Perez-Perez Gl, Chen J, HerreraGoepfert R, Chihu-Amparan L, Flores-Luna L, Mohar-Betancourt A, LopezCarrillo L. Dietary folate and vitamin B12 intake before diagnosis decreases gastric cancer mortality risk among susceptible MTHFR 677TT carriers. Nutrition. 2010:26:201-8.

10. Xu X, Gammon MD, Zeisel SH, Bradshaw PT, Wetmur JG, Teitelbaum SL, Neugut Al, Santella RM, Chen J. High intakes of choline and betaine reduce breast cancer mortality in a population-based study. FASEB J. 2009;23:4022-8.

11. Alan L, Buchman MEA, Sohel M, Dubin M, Jenden DJ, Roch M, et al. Choline Deficiency Causes Reversible Hepatic Abnormalities in Patients Receiving
Parenteral Nutrition: Proof of a Human Choline Requirement: A PlaceboControlled Trial. J Parenter Enter Nutr. 2001:25:260-8.

12. CJ MN, Hay SM, Rucklidge GJ, Reid M, Duncan G, Maloney CA, Rees WD. Disruption of lipid metabolism in the liver of the pregnant rat fed folatedeficient and methyl donor-deficient diets. Br J Nutr. 2008;99(2):262-71.

13. Sherriff JL, O'Sullivan TA, Properzi C, Oddo JL, Adams LA. Choline, Its Potential Role in Nonalcoholic Fatty Liver Disease, and the Case for Human and Bacterial Genes. Adv Nutr. 2016;7(1):5-13.

14. Mato JM, Lu SC. The hepatocarcinogenic effect of methionine and choline deficient diets: an adaptation to the Warburg effect? Alcohol Clin Exp Res. 2011:35(5):811-4.

15. Erina KY, Maki H, Tomoyuki N, Takashi H. Accumulation of Liver Lipids Induced by Vitamin B6 Deficiency Was Effectively Ameliorated by Choline and, to a Lesser Extent, Betaine. J Nuti Sci Vitaminol. 2019;65:94-101.

16. Kitagawa E, Yamamoto T, Fujishita M, Ota Y, Yamamoto K, Nakagawa T, Hayakawa T. Choline and betaine ameliorate liver lipid accumulation induced by vitamin B6 deficiency in rats. Biosci Biotechnol Biochem. 2017;81:316-22.

17. Jiang $X$, Greenwald $E$, Jack-Roberts $C$. Effects of choline on DNA methylation and macronutrient metabolic gene expression in in vitro models of hyperglycemia. Nutr Metab Insights. 2016;9:11-7.

18. Butler LM, Arning E, Wang R, Bottiglieri T, Govindarajan S, Gao YT, Yuan JM. Prediagnostic levels of serum one-carbon metabolites and risk of hepatocellular carcinoma. Cancer Epidemiol Biomarkers Prev. 2013;22:1884-93.

19. Liu Z-Y, Tan X-Y, Li Q-J, Liao G-C, Fang A-P, Zhang D-M, Chen P-Y, Wang XY, Luo Y, Long J-A, Zhong R-H, Zhu H-L. Trimethylamine N-oxide, a gut microbiota-dependent metabolite of choline, is positively associated with the risk of primary liver cancer: a case-control study. Nutr Metab. 2018;15:81.

20. Zhu HL, Huang J, Xu M, Fang YJ, Lu MS, Pan ZZ, Huang WQ, Chen YM, Zhang CX. Higher dietary intakes of choline and betaine are associated with a lower risk of primary liver cancer: a case-control study. Sci Rep. 2017;117:839-50.

21. Zeng FF, Xu CH, Liu YT, Fan YY, Lin XL, Lu YK, Zhang CX, Chen YM. Choline and betaine intakes are associated with reduced risk of nasopharyngeal carcinoma in adults: a case-control study. Br J Cancer. 2014;110:808-16.

22. Fang AP, Chen PY, Wang XY, Liu ZY, Zhang DM, Luo Y, Liao GC, Long JA, Zhong RH, Zhou ZG, Xu YJ, Xu XJ, et al. Serum copper and zinc levels at diagnosis and hepatocellular carcinoma survival in the Guangdong liver Cancer cohort. Int J Cancer. 2019:144(11):2823-32.

23. Fang AP, Liu ZY, Liao GC, Chen PY, Wang XY, Zhang DM, Luo Y, Long JA, Zhong RH, Zhou ZG, Xu YJ, Xu XJ, et al. Serum folate concentrations at diagnosis are associated with hepatocellular carcinoma survival in the Guangdong liver Cancer cohort study. Br J Nutr. 2019;121(12):1-38.

24. Al B, Benson TAA III, Edgar Ben-Josef P, Bloomston M, Botha JF, Clary BM, et al. Hepatobiliary cancers: clinical practice guidelines in oncology. J Natl Compr Cancer Net. 2009:7:350-91.

25. Cillo U, Vitale A, Grigoletto F, Farinati F, Brolese A, Zanus G, Neri D, Boccagni P. Srsen N, D'Amico F, Ciarleglio FA, Bridda A, et al. Prospective validation of the Barcelona clinic liver Cancer staging system. J Hepatol. 2006;44:723-31.

26. Mehedint MG, Zeisel SH. Choline's role in maintaining liver function: new evidence for epigenetic mechanisms. Curr Opin Clin Nutr Metab Care. 2013;16:339-45.

27. Wiedeman AM, Barr SI, Green TJ, Xu Z, Innis SM, Kitts DD. Dietary choline intake: current state of knowledge across the life cycle. Nutrients. 2018;10:1513.

28. Pellanda $\mathrm{H}$. Betaine homocysteine methyltransferase (BHMT)-dependent remethylation pathway in human healthy and tumoral liver. Clin Chem Lab Med. 2013;51:617-21.

29. Jin B, Gong Z, Yang N, Huang Z, Zeng S, Chen H, Hu S, Pan G. Downregulation of betaine homocysteine methyltransferase (BHMT) in hepatocellular carcinoma associates with poor prognosis. Tumour Biol. 2016:37:5911-7.

30. Oliva J, Zhong J, Buslon VS, French SW. The effect of SAMe and betaine on Hepa 1-6, C34 and E47 liver cell survival in vitro. Exp Mol Pathol. 2012;92: $126-30$.

31. Sieghart W, Pinter M, Hucke F, Graziadei I, Schoniger-Hekele M, Muller C, Vogel W, Trauner M, Peck-Radosavljevic M. Single determination of Creactive protein at the time of diagnosis predicts long-term outcome of patients with hepatocellular carcinoma. Hepatology. 2013;57:2224-34.

\section{Publisher's Note}

Springer Nature remains neutral with regard to jurisdictional claims in published maps and institutional affiliations. 